\title{
Féeries
}

Études sur le conte merveilleux, XVII $-\mathrm{XIX}{ }^{\mathrm{e}}$ siècle

\section{Wieland ou le triomphe de la nature sur la féerie dans Les aventures de Don Sylvio von Rosalva (1764)}

Wieland, or the Triumph of Nature over the Marvellous in Don Sylvio von Rosalva's Adventures (1764)

\section{Alain Montandon}

\section{Édition électronique}

URL : http://journals.openedition.org/feeries/1842

DOI : $10.4000 /$ feeries. 1842

ISSN : $1957-7753$

Éditeur

UGA Éditions/Université Grenoble Alpes

Édition imprimée

ISBN : 978-2-37747-075-4

ISSN : 1766-2842

\section{Référence électronique}

Alain Montandon, « Wieland ou le triomphe de la nature sur la féerie dans Les aventures de Don Sylvio von Rosalva (1764) », Féeries [En ligne], 15 | 2018, mis en ligne le 14 février 2019, consulté le 08 septembre 2020. URL : http://journals.openedition.org/feeries/1842 ; DOI : https://doi.org/10.4000/ feeries. 1842

Ce document a été généré automatiquement le 8 septembre 2020

(c) Féeries 


\title{
Wieland ou le triomphe de la nature sur la féerie dans Les aventures de Don Sylvio von Rosalva (1764)
}

\author{
Wieland, or the Triumph of Nature over the Marvellous in Don Sylvio von \\ Rosalva's Adventures (1764)
}

\section{Alain Montandon}

Christoph Martin Wieland publie en 1764 un roman qui passe pour être le premier roman moderne de la littérature allemande. Le titre complet, Le triomphe de la nature sur la rêverie ou les aventures de Don Sylvio de Rosalva ${ }^{1}$, indique le conflit fondamental de l'imagination et de la réalité objective.

1 Le jeune héros, lecteur féru de contes, imagine vivre en féerie, transposant en imagination ses perceptions du réel et interprétant tout ce qui l'entoure et tout ce qui lui arrive suivant le monde des contes (ceux des conteurs français largement diffusés en Allemagne, et tout particulièrement ceux de Madame d'Aulnoy).

2 Pour le désenchanter ses amis lui racontent un conte merveilleux, le conte de Biribinker, dont l'extravagance est destinée à le ramener à la saine réalité. À une époque où le conte est déjà devenu depuis longtemps une forme parodique de luimême, Wieland écrit la parodie de la parodie avec le résultat que celle-ci est aussi l'un des meilleurs récits féeriques qui soit dans le genre. En distinguant alors réalité empirique et monde de l'imagination, Don Sylvio renonce à ses folies, s'ouvre au monde et à l'expérience.

3 Le jeu de l'illusion et de ses ruptures, la critique de la superstition, les rapports du perçu et de l'imaginé, tout en déployant tout un éventail de sensations réelles et imaginaires dont s'amuse le savant auteur des Lumières (inspiré de Cervantès comme de Lesage), alternant l'ivresse d'un rococo fabuleux avec le dégrisement de la raison, constituent le charme de ce texte exemplaire dont le romanesque très $\mathrm{xVIII}^{\mathrm{e}}$ siècle se nourrit du monde de la féerie. 
Il s'agit par l'intermédiaire du conte de fées de faire la critique rationaliste des formes de la superstition et de la rêverie (Schwärmerei). C'est, écrit Wieland à Gessner (5 août 1763), « une sorte de satire. Un roman qui sous l'apparence de la frivolité, est assez philosophique ${ }^{2}$ » où fiction et réel ne cessent de s'entremêler.

\section{Des dangers de la lecture}

Le fanatisme (Schwärmerei) dont est victime Don Sylvio provient de son éducation solitaire dans un château coupé du monde, et entièrement nourrie par une bibliothèque riche de façon exclusive des contes de fées français dont le jeune homme possède une érudition exemplaire qu'il utilise pour interpréter le monde extérieur. En cela, ce type d'éducation est opposée à la conception de l'homme des Lumières qu'est Wieland qui ne cesse de déployer dans son texte une culture cosmopolite, faisant largement appel aux écrivains et penseurs de l'Antiquité gréco-latine, à la littérature espagnole avec Cervantès, à la littérature française avec Lesage et les auteurs des contes de fées, aux romanciers anglais et aux écrivains italiens. Cette grande culture qui permet de confronter les idées et s'assurer d'un vif esprit critique est contraire à une vision unique, refermée sur elle-même et source de toutes les superstitions et préjugés. Wieland est ainsi amené à suggérer l'étroitesse d'esprit de l'éducation donnée par l'église catholique qui pervertit le jugement par un dogmatisme éloigné de toute réflexion et opposé au bon sens et à la raison. Aussi la réflexion " assez philosophique » que suggère Wieland est-elle toujours d'actualité quand on songe aux enseignements religieux donnés de nos jours, dans les écoles coraniques par exemple, enseignements qui loin de tout esprit critique, conduisent logiquement au fanatisme. L'intérêt actuel de ce roman est de démontrer la perversion d'un endoctrinement par la lecture et comment les amis de Don Sylvio se livrent à une véritable entreprise de « déradicalisation».

5 Si Donna Mencia, la tante de Don Sylvio, n'a que mépris pour les contes, «petits jeux d'esprit qu'on n'écrit que pour occuper les enfants ou pour égayer les vieillards ${ }^{3}$ », si le père de Don Sylvio convenait que tout en l'amusant ce n'étaient que des frivolités, il convient de remarquer que le jeune homme fut amené à leur lecture par une espèce de nonchalance, voire par une certaine paresse d'esprit, car la brièveté de chaque histoire le prévint extrêmement en faveur des contes qu'il se mit à dévorer. On voit par là que Wieland a une attitude ambiguë vis-à-vis des contes. Sans doute est-il comme Don Sylvio, dégoûté des lectures d'in-folio d'une épaisseur prodigieuse qui avaient les faveurs de la tante. Sans doute est-il lui-même grand amateur de contes comme le prouve sa grande connaissance des recueils de contes et lui-même s'attache à en écrire, non sans malice comme on le voit avec l'invention du conte de Biribinker et bien d'autres ${ }^{4}$. Mais dans le même temps, les contes ne sont plus guère pris au sérieux, tant ils ont fait l'objet de parodies et d'autoparodies. S'ils peuvent encore avoir des fonctions didactiques, satiriques et morales, ils restent loin des fonctions poétiques fort sérieuses que le romantisme va leur attribuer.

6 Le terme de Schwärmerei dans le titre du roman, qui a pu être traduit en français par «rêverie » ou « exaltation », devrait plutôt l'être par «fanatisme », car cette "rêverie » est bien une espèce de délire loin de toute réalité. Le rêveur est un être qui s'absente du monde pour errer dans celui de son imagination et de sa fantaisie. Wieland a lui-même fort bien précisé comment il fallait entendre le terme de Schwärmerei. Dans le 
«Supplément de l'éditeur» ajouté par lui-même à l'article de Leonhard Meister, Auszüge aus einer Vorlesung über die Schwärmerey paru dans Der teutsche Merkur en 1775, Wieland jugeant que pour beaucoup les notions d'enthousiasme et de Schwärmerei sont identiques, s'emploie à les distinguer :

Je nomme [...] exaltation (Schwärmerei) un échauffement de l'âme par des objets qui ne sont absolument pas dans la nature, ou du moins qui ne sont pas ce pour quoi l'âme enivrée les tient [...] Au mot Schwärmerei, pris en ce sens, correspond assez exactement le mot Fanaticismus [...] $]^{5}$.

7 Il ajoute que ce fanatisme a aussi été attribué à une sorte d'exaltation religieuse. On a pu rapprocher l'étymologie de Schwärmer de Schwarm, «l'essaim », sous-entendant ainsi que l'esprit du "rêveur exalté » se caractérise par un penchant certain à " essaimer ", c'est-à-dire par un prosélytisme assumé, et il n'est pas anodin de rappeler que le terme allemand Schwarm désigne aussi les sectes au XvI ${ }^{\mathrm{e}}$ siècle, ainsi que le note Charlotte Coulombeau ${ }^{6}$. Il est intéressant de voir comment Pedrillo, le valet de Don Sylvio, en vient à partager pour une part l'exaltation et les illusions de son maitre et, bien qu'il soit souvent plus critique et contradicteur, il se laisse parfois entraîner même au-delà des rêveries de son maître.

Cependant, si l'enthousiasme est valorisé, « le fanatisme est maladie de l'âme, véritable fièvre de l'âme ${ }^{7}$ ", car dominé par des représentations illusoires.

9 Le grand débat qui anime les protagonistes principaux, Don Sylvio et Pedrillo, est de savoir ce qui permet de faire la différence entre une appréhension véritable et une appréhension illusoire du réel. L. Meister dans son essai disait que la plupart des hommes sont plus ou moins des Don Quichotte et que, déçue par la réalité, jugeant insuffisant ce que les yeux donnent à voir, l'âme se donne les verres magiques de l'imagination (Zauberglase der Einbildunsgkraft). Il nommait la difficulté qui ne cesse de tourmenter nos deux héros : « Sans aucun doute les frontières entre l'erreur et la vérité ne sont pas moins difficiles à déterminer que les frontières entre l'ombre et la lumière, le sommeil et l'éveil's.»

\section{L'instinct du merveilleux : le moment féerie}

Les Lumières ont combattu avec ardeur toutes les formes de superstition. Wieland, loin de déroger à l'entreprise critique, combat avec ardeur préjugés et croyances. Dans Über den Hang der Menschen an Magie und Geistererscheinungen zu glauben, il analyse de manière anthropologique la croyance profondément enracinée en l'homme au merveilleux et aux êtres surnaturels. L'explication est historique, car à l'origine les peuples eurent besoin d'animer d'esprits ce qui les entourait, mais aussi anthropologique puisque la peur de la mort est également à l'origine de croyances irrationnelles. Ce besoin de merveilleux est qualifié de faiblesse humaine (die schwache Seite der menschlichen Natur) contre laquelle il convient de lutter fermement par l'expérience et la raison'.

Aussi le combat doit-il être perpétuel contre les impulsions de l'imagination, d'autant plus que celle-ci est fortement ancrée en l'homme et qu'elle lui est aussi une source de plaisir. Dans la préface à son recueil de contes, Dschinnistan, il écrit :

Il paraît étrange que deux penchants si contradictoires comme l'inclination au merveilleux et l'amour du vrai soient équivalents pour l'homme, même essentiels et pourtant ce n'est pas autrement ${ }^{10}$. 
11 Wieland pense que la rêverie et la superstition étendent leur influence sur toutes les branches de la vie humaine, et cela en raison du fait que la rêverie est une exacerbation de la subjectivité et de la passivité de notre nature. La tolérance, l'activité critique et rationnelle, la désillusion par l'usage de la plaisanterie et une bonne humeur ${ }^{11}$ doivent contrebalancer cette tendance qui, sinon, prend vite des formes pathologiques. Wieland veut ridiculiser «la sotte crédulité du peuple dans la personne de Pedrillo, et en général ce que Juvénal appelle veteres avias ${ }^{12} »$.

De là vient que les paysans, dont le travail ne leur laisse pas le loisir de tirer des connaissances claires des différentes impressions que la nature fait sur eux, sont, en général, plus superstitieux que d'autres. De la confusion de leurs idées, naît leur croyance aux chasses invisibles dans les bois, aux fées qui dansent la nuit dans les prairies, aux génies bienfaisants ou méchants, au cauchemar qui tourmente les filles, aux nixes, aux esprits follets, et à je ne sais combien d'autres fantômes dont ils racontent nombre d'histoires et dont la réalité est selon eux, une chose si avérée qu'on n'oserait la nier, à moins de passer aux yeux de la plupart pour sot ou impie ${ }^{13}$.

Cela permet d'expliquer les visions fantasques et les superstitions du valet qu'il traite d'esclave de la superstition et dont la tête est farcie des récits d'aventures, d'exploits des chevaliers, des fées, des créatures de la mythologie antique et des histoires populaires qui pêle-mêle alimentent, avec les nombreux proverbes qu'il tient de sa grand-mère, un comportement irrationnel.

Le mysticisme et la superstition étendent leur influence sur tous les rameaux de l'activité humaine, car l'un et l'autre sont naturels à l'homme; mais à toutes les époques on a pensé qu'il était nécessaire et sain de se moquer de ces ressorts de la nature humaine. La plaisanterie et l'ironie, accompagnant le bon usage de nos cinq sens, ont toujours constitué le meilleur remède à ces égarements et c'est dans cette intention, comme l'indique le titre, que l'histoire de Don Sylvio a été écrite ${ }^{14}$.

Le roman qui, sous l'apparence de la frivolité est assez philosophique, n'est pas que cela.

\section{Psychanalyse des contes de fées}

Don Sylvio en effet est non seulement un roman d'aventure, mais également un roman d'initiation à un amour véritable et non plus fantasmé. Du moins est-ce ainsi que le lecteur est amené à comprendre le récit. Cependant, la dimension fantasmatique de l'amour est fort bien représentée, puisque Don Sylvio aime une femme semblable à ses fantasmes et Donna Felicia, grande lectrice nourrie de fantaisies romanesques, de bergers d'Arcadie, se plaît à retrouver dans Don Sylvio un Céladon de l'Astrée.

Un roman d'initiation à l'image des contes, car l'aventure du jeune homme ressemble à ces féeries qu'il dévore dans la bibliothèque de son père. Le schéma bien connu de Propp $^{15}$ s'applique aussi au déroulement de l'histoire : éloignement, transgression de l'interdit, obstacles, animal auxiliaire (le petit chien Pimpimp), rencontre du personnage antagoniste de la sorcière bohémienne, etc. Autrement dit, toute l'histoire peut, elle aussi, être lue comme un conte. Mais aussi comme un roman de formation. Il n'est pas inutile de rappeler que Wieland, à la même époque, est en train d'écrire Agathon (première édition en 1766) qui sera le premier grand modèle du Bildungsroman de la littérature de langue allemande.

Le jeune Agathon est un jeune homme, un rêveur (Schwärmer) enthousiaste, féru d'idéaux qui ne correspondent pas à la réalité qu'il va progressivement découvrir. 
Agathon est élevé en secret au temple de Delphes où il reçoit une éducation très pieuse. Initié aux mystères orphiques il traverse d'abord une période d'exaltation mystique, fort proche de la période piétiste de Wieland, puis après avoir découvert la doctrine pythagoricienne, puis la philosophie de Platon, il porte un pur amour à Psyché mais, séparé de celle-ci, il connaît maintes aventures qui mettent à mal son idéalisme, dans les bras de Daphné qui l'initie à la volupté des sens, et par le biais de la philosophie matérialiste et sensualiste d'Hippias qui condamne le sentimentalisme rêveur d'Agathon. On voit combien ce sont les mêmes interrogations qui sont posées dans Don Sylvio entre matérialisme et idéalisme.

15 Tout comme Agathon, Don Sylvio est confronté aux obstacles de la réalité, de ce que Wieland nomme la nature ( Natur»), pour finalement trouver un équilibre plus harmonieux et satisfaisant, conciliant le désir et sa réalisation. Car il s'agit bien de désir et de libido. De même que Heinrich von Ofterdingen, dans le roman éponyme de Novalis, exprimera son insatisfaction en s'agitant sur sa couche (Der Jüngling lag unruhig auf seinem Lager!) et par la satisfaction du désir dans le rêve de la fleur bleue (dont Bachelard a bien dit qu'elle était rouge !), le jeune Don Sylvio trouve dans les livres de féerie une image où projeter son désir, et il poursuit dans ses rêves des "pensées chimériques et des songes agréables venant seconder ses désirs ». "Il y a une certaine fièvre à laquelle l'âme humaine est fort sujette dès la quatorzième année de son âge » note de manière tout à fait médicale l'auteur ${ }^{16}$. On ne s'étonnera pas que notre jeune homme recule devant la femme qu'on veut lui faire épouser, qu'il trouve d'autant plus laide qu'elle le presse à conclure. Lorsque tous deux se promènent au clair de lune et que la nature assoupie étend un charme délicieux de volupté, Don Sylvio se laisse prendre par la sensualité ambiante et la pression d'une main féminine. Nageant dans le sublime, il oublie l'identité de la fille jusqu'à un point où défenses et résistances, face à un objet trop concret, provoquent chez lui rejet et dégoût. À la place de la charmante Princesse qu'il a cru embrasser, il ne voit plus qu'une naine horrible dont les embrassements lui sont dégoûtants.

Mais soit que l'imagination de notre héros fut épuisée par la quantité de galimatias qu'il avait débitée à sa chère princesse supposée, ou qu'il n'y eut d'illusion, d'extravagance ou d'enchantement assez fort pour tenir contre l'aspect de Donna Mergelina, ils étaient à peine sortis du bosquet et l'on y voyait un peu plus clair, que Don Sylvio à peine eut-il jeté les yeux sur sa compagne qu'il poussa un grand $\mathrm{cri}^{17}$.

Nous n'ignorons pas qu'il s'agit là d'un topos récurrent dans la littérature, à commencer par Cazotte où dans La Belle par accident on trouve une semblable situation. Si le jeune homme est trompé par la blancheur d'une main fardée à dessein, il sera cependant désillusionné par l'odeur de la vieille. Pourtant les forces de l'imagination ont un pouvoir qu'on pourrait qualifier de pygmalionesque, puisqu'elles permettent de donner corps à l'objet du désir. Les sensualistes, tel Condillac, pensent que l'imagination " procure des jouissances qui, à certains égards, l'emportent sur la réalité même : car elle ne manque pas de supposer dans les objets dont elle fait jouir, toutes les qualités qu'on désire y trouver ${ }^{18} »$. Condillac définit l'imagination comme cette mémoire vive, qui fait paraître présent ce qui est absent. Un écrivain sensualiste comme Meusnier de Querlon imagine fort bien dans son conte, Les Dortoirs de Lacédémone $e^{19}$, une telle situation où des rivales jalouses glissent à son insu dans le lit de Glycon amoureux d'une jeune Messénienne, une vieille femme :

Figurez-vous un jeune homme ivre d'amour et fortement préoccupé de l'objet qu'il croit tenir entre ses mains : je vous laisse imaginer toutes les délices qu'il dut faire 
goûter à cette vieille (pour peu qu'il lui restât de sentiment) et celles qu'il ressentit lui-même. Dans la douce illusion où il était plongé, dans le délire de ses sens, le fantôme palpable lui représente tout ce qu'il imagine dans la Messénienne ${ }^{20}$. perceptions. Un papillon est bien pour lui un papillon : c'est dans l'interprétation de ce qu'il perçoit que se joue le moment féerique. Il en est de même avec Pedrillo qui s'interroge sur la réalité de sa vision ; pour cela il interroge son corps et ses sens :

Est-ce que je m'imagine que je veille, ou vois-je bien de mes propres yeux? Un petit moment, on va savoir, je vais me pincer les joues et les mollets - bon, bon, je suis bien moi-même cela est sûr ! - ce sont mes yeux ! J'ai beau les frotter tant que je veux, ils me font toujours voir ces deux belles créatures, si elles en sont; mais je suis pleinement persuadé que ce sont des fées et des plus belles ${ }^{21}$.

Les rêves érotiques eux-mêmes sont sujets à de cruelles censures et, lorsqu'il veut embrasser la chère amante de ses rêves, il s'aperçoit (toujours en rêve) avec horreur " que la figure, qu'il pressait tendrement sur son sein, était celle de la grosse Maritorne, servante de sa basse-cour. Ces lèvres qui, un instant auparavant, semblaient exhaler le nectar et l'ambroisie, sentaient si puissamment l'ail et le fromage qu'il y avait de quoi faire mourir de dégoût et d'aversion ${ }^{22}$ ». Il est clair que le jeune homme est pris entre une libido et une défense, effet d'un sentiment de culpabilité et de l'appréhension à réaliser un désir qu'il méconnaît et qui l'effraie. La censure s'incarne dans des figures maléfiques, comme celle du Nain-vert ou de la fée Franfreluche : "Il envisageait ses songes comme de très mauvais présages. Il ne doutait pas du tout qu'il n'eût une ennemie puissante, attentive à traverser les sentiments qu'il avait conçus pour la charmante inconnue qu'il devait aimer ${ }^{23}$.» Cette ennemie invisible et fort puissante sera partiellement vaincue par le conte de Biribinker qui développe l'irrésistible condescendance du jeune héros à embrasser toutes les aventures amoureuses qui se présentent à lui. Cette liberté et ce libertinage offrent un plaisant tableau des plaisirs des sens et du sexe aux fins d'éduquer Don Sylvio.

première transgression est sans aucun doute celle des lectures que le sérieux et la morale de sa tante lui avaient interdites. Il est d'ailleurs fort conscient que ce genre de lecture est répréhensible ${ }^{24}$ :

Un certain instinct qui apprend aux jeunes gens les moins expérimentés ce qu'ils doivent avouer ou taire à ceux qui les gouvernent, avertit Don Sylvio qu'il ne fallait pas laisser découvrir à sa tante la trouvaille qu'il avait faite. La réserve qu'il était obligé d'avoir, ne faisait qu'augmenter sa tendresse pour les fées, et son goût pour les contes ${ }^{25}$.

Il s'enfuit avec le volume qu'il cache sous son chevet pour le lire et le relire à l'écart, une répétition qui fait de la lecture une activité bien solitaire au plaisir de laquelle il se livre entièrement, au point de s'identifier pleinement à ses découvertes: "Son imagination saisissait avec facilité tous les détails que lui présentait son livre: il ne lisait pas; il voyait, il entendait, il sentait ${ }^{26}$." C'est dire que l'investissement dans un merveilleux offre un dérivatif à la solitude et à l'ennui : «L'imagination est occupée à remplir le vide que l'uniformité des objets qui s'offrent au sens, laisse dans l'âme ${ }^{27}$. " Pour reprendre l'expression d'Évelyne Jacquelin, la lecture ouvre à l'adolescent de dixsept ans « l'espace enchanteur et périlleux du désir ${ }^{28}$ ».

21 À partir de là, Don Sylvio modèle son environnement, ses pensées et toute son existence en suivant un scénario féerique dominant toutes ses interprétations. C'est ce "mécanisme d'ajustement fantasmatique ${ }^{29}$ ", avec lequel son valet Pedrillo entre en 
contradiction, qui l'empêche d'aborder clairement une réalité qui sans cesse se rebelle à ses vues et qu'il s'efforce sans cesse de redresser par des interprétations suivant la grille féerique. La rencontre de Felicia le place dans une situation aporétique entre la personne réelle et son image, titre du chapitre VIII du cinquième livre : Combat entre l'amour pour le portrait, et celui pour l'original.

De l'issue de ce conflit dépendra évidemment la guérison de sa folie qui l'amènera enfin à ne plus considérer sa vie passée que comme un vain songe.

\section{Libertinage homéopathique}

L'Histoire du Prince Biribinker est une facétieuse parodie des contes de fées français du $\mathrm{xVIII}^{\mathrm{e}}$ siècle. Le bon magicien Karamussal a donné au prince le nom de Biribinker pour le protéger de mauvais enchantements. Élevé dans une ruche par une abeille :

Le Prince grandit et surpassa en beauté et en rares qualités tout ce qu'on n'avait jamais vu. Il ne crachait que du sirop, ne pissait que de l'eau de fleurs d'orangers, et ses langes contenaient des choses si délicieuses, qu'on les envoyait de temps en temps à la Reine pour en compléter son dessert aux jours de fêtes ${ }^{30}$.

Il ne fallait cependant pas lui laisser voir une laitière avant l'âge de dix-huit ans. Il s'ensuit des aventures plus farfelues les unes que les autres, de ces aventures que l'on ne trouve que dans les contes de fées, avec la rencontre de chèvres bleues attachées à une crèche de diamants, ou de chèvres couleur de rose que trait une ravissante laitière qui fuit le héros en entendant son nom. Amoureux de cette Galactine, il doit cependant affronter un géant, rencontrer des poissons chantant, une énorme baleine dans le ventre de laquelle il découvre un palais invisible construit de flammes par des salamandres, et faire connaissance de sylphes, d'ondines, de gnomes et de la fée Cristalline qu'il délivre de l'enchanteur Padmanaba.

L'éducation du jeune prince dans les contes français parodiques (que ce soit chez Hamilton, Duclos, Crébillon, etc.) était présentée chez La Morlière, dans Angola, avec une élégance badine: il apprend que le plaisir systématique prescrit par la fée Lumineuse $^{31}$ est la source d'un bonheur qui réside dans la "variété des plaisirs ", dans l'inconstance, dans "tous les agréments" qui font fi de toute fidélité. Le monde d'Angola est celui de la galanterie érotique teintée d'humour, celui des nudités surprises au bain, celui des initiations féminines ${ }^{32}$ qui invitent en papillonnant le héros dans des séjours enchanteurs: "Tout invitait à l'amour dans ce séjour dangereux. L'ameublement inventé par la mollesse portait un caractère de volupté difficile à rendre; beaucoup de glaces, des peintures tendres et sensuelles, une duchesse, des bergères, des chaises longues, semblaient tacitement désigner l'usage auquel elles étaient destinées $[. . .]^{33}$.» Rien n'échappe à cette atmosphère de séduction («Elle était couchée négligemment, et par décence faisait des nœuds. Son déshabillé galant et léger laissait voir une partie de ses charmes et semblait ne cacher l'autre que pour augmenter les désirs ${ }^{34} »$ ) faite pour former le jeune Angola à cette physique amoureuse qui veut que ce soit la «sympathie d'organes établie qui les [les amants] éclaire sur leurs sentiments mutuels ${ }^{35}$ ». Ce Corteggiano de l'éducation amoureuse est d'un raffinement très poussé et d'une esthétique rococo faite pour combattre l'ennui. Wieland reprend de façon identique cette éducation érotique chez son héros, partagé entre un amour idéal pour la laitière et des nuits d'intense passion sensuelle, ce que la fée Mirabelle lui reproche : «A-t-on jamais vu un amant fait comme vous? Vous rôdez pendant toute la journée pour chercher partout votre bien-aimée, et puis vous passez la 
nuit entière dans les bras d'une autre; le lendemain votre amour recommence, et le soir votre infidélité de même ${ }^{36}$.»

Émerveillé par la vue de la beauté féerique d'un palais aux murs couverts de glaces de cristal, étourdi par des odeurs délicieuses apportées par les zéphyrs, Biribinker découvre pour sa gouverne :

Un lit de repos voluptueux, dont un petit dieu d'amour de marbre qui paraissait respirer, ôtait tant soit peu les rideaux flottants, était le seul meuble dans cet appartement charmant; il fît naître dans le cœur de notre Prince un désir mystérieux après certaines choses, dont, aussi novice qu'il était encore, il n'eût que des idées confuses, quoique la tapisserie qu'il considéra fort attentivement et non sans quelque douce inquiétude, lui fit voir un tant soit peu clair dans cette affaire ${ }^{37}$.

Wieland qui goûtait l'impertinence équivoque de Hamilton, de Duclos et de Crébillon en composant ce qui, de fait, est l'un des tout premiers contes littéraires en Allemagne, imite les fantaisies libertines propres à la féerie. Biribinker trouve en effet un pot de chambre (certes en cristal) duquel il fait usage, ce qui a pour conséquence de délivrer une jeune nymphe qui avait été métamorphosée à sa grande honte. Après un repas composé de mets délicieux, le prince était :

trop poli pour laisser se coucher seule sur le sopha une si belle fée; et la belle fée était trop reconnaissante pour lui refuser la compagnie [...]. En un mot la politesse d'un côté, et la reconnaissance de l'autre furent poussées aussi loin que possible, et Biribinker se montra parfaitement digne de la bonne idée que Cristalline avait conçue pour lui dès le premier moment ${ }^{38}$.

Tout ce conte est une apologie des sens bien faite pour guérir de la rêverie sentimentale éthérée. Tous les sens sont mis en éveil pour donner à Biribinker «les sensations les plus agréables » et l'amener à des situations sans équivoque qui faisaient dire à Moritz August von Thümmel que « celui qui lit Wieland est un $\operatorname{coquin}^{39}$ ", mais cela n'a pas empêché la duchesse Anna Amalia à Weimar de confier l'éducation du prince Karl August à l'auteur de l'Histoire du prince Biribinker, qui ne s'en est pas trop mal tiré ! « Le Biribinker est un conte extravagant, une débauche d'esprit dont le but était de faire rire toute l'espèce humaine et de turlupiner certaines femmes qui osent prétendre au sentiment et ne sont au fond que je les ai dépeintes à la Hogarth, mais où est le mal ? $[s i c]^{40} . »$

Don Sylvio est brusquement désenchanté quand il apprend que l'histoire de Biribinker, racontée par Don Gabriel à des fins homéopathiques puisqu'il veut guérir le poison par le poison, est purement inventée par son narrateur. En distinguant alors réalité empirique et monde de l'imagination, Don Sylvio renonce à ses folies, s'ouvre au monde et à l'expérience. Wieland applique ici les principes d'une psychologie empirique ${ }^{41}$. Don Sylvio finit par céder devant une réalité qui vient effacer les images de ses fables imaginaires :

Je vous dis ce que je sens, et je voudrais pouvoir vous le dire dans un langage qui exprimât mes vrais sentiments. Ce que je sens depuis que je vous vois, diffère infiniment des délires d'une imagination échauffée. Votre premier aspect a éteint tout le feu de mon imagination; je ne me souviens de ma vie passée que comme d'un vain songe; ce n'est que depuis ce moment fortuné, où je vous vis pour la première fois, que ma vraie vie a commencé ${ }^{42}$.

Si la «rêverie " (Schwärmerei) est « un échauffement de l'âme à partir d'objets qui ne sont pas dans la nature " ainsi que l'affirme Wieland ${ }^{43}$, on comprend que la question $\mathrm{du}$ statut même de la fiction narrative soit posée, de sa crédibilité et du crédit dans lequel le lecteur doit la tenir. Les formes parodiques en soumettant la fiction au fameux « test 
du ridicule » servent à souligner l'extravagance de l'imagination, et par contrecoup une indispensable référentialité. Critique et parodie des "rêveries" ne cessent de poser le problème du réel. L'histoire de Don Sylvio n'est ni vraie ni vraisemblable, c'est un amusement de l'esprit qui donne à penser. «En feignant de badiner et de faire le fou, on philosophe mieux que Chrysippe et que Cléante ${ }^{44}$. »

Le conte de fées d'un Biribinker mettant en abyme le roman lui-même pose de manière moderne le rapport des mondes possibles et du réel. Aussi le don Quichottisme de ce conte philosophique rococo connut un succès mérité.

\section{NOTES}

1. Le premier volume parut début mars 1764 et le second volume en avril de la même année, chez le libraire Fr. Bartholomai à Ulm, sous le titre Der Sieg der Natur über die Schwärmerei, oder die Abenteuer des Don Sylvio von Rosalva. Eine Geschichte, wo alles Wunderbare natürlich zugeht (édition de référence de notre traduction, à paraître, voir ci-dessous). En France, Les Aventures merveilleuses de Don Sylvio de Rosalva a été traduit par K. S. Walther à Dresde et Paris en 1769 (réédité en 1772), puis repris sous forme d'extrait dans la Bibliothèque universelle des romans (janvier 1785), tandis que la traduction de Madame d'Ussieux trouve sa place dans le Cabinet des fées (t. XXXVI) en 1786. Cette dernière traduction intitulée d'abord Le Nouveau Don Quichotte, imité de l'allemand de M. Wieland (1770) est d'une grande liberté. Il s'agit moins d'une traduction que d'une adaptation. "On a tâché de faire un ouvrage Français d'une production Allemande » écrit Madame d'Ussieux qui insiste beaucoup sur la parenté avec Cervantes: «Il me semble que don Silvio pourrait être une espèce de Don Quichotte qui, pour me servir de l'expression de Pédrillo, voyage en Féerie, comme faisait autrefois le Chevalier de la Manche. » Voir notre édition Les aventures de Don Sylvio von Rosalva, ou La victoire de la nature sur la rêverie, trad. fr. et éd. critique par A. Montandon, Paris, Classiques Garnier, 2019.

2. Ausgewählte Briefe von C.M. Wieland an verschiedene Freunde, Zürich, 1815, II, p. 220-221.

3. C. M. Wieland, Don Sylvio, Premier livre, chap. 4.

4. Wieland est l'auteur de nombreux contes (voir en particulier Dschinnistan, oder auserlesene Feenund Geister-Mährchen), dont certains furent très rapidement traduits en français. Voir par exemple Contes comiques traduits de l'allemand, Francfort, 1771 ; Contes de Wieland et du baron de Ramdohr, Paris, 1813, 2 t.

5. «Ich nenne [...] Schwärmerey eine Erhitzung der Seele von Gegenständen die entweder gar nicht in die Natur sind, worüber die betauschte Seele sie ansieht. [...] Dem Worte Schwärmerey, in dieser Bedeutung genomment, entspricht das Wort Fanaticismus ziemlich genau [...]. » (Der teutsche Merkur, 1775, 4. Vierteljahr, p. 152)

6. Ch. Coulombeau, "Sur un problème qui vient du temps", Les Études philosophiques, $\mathrm{n}^{\circ} 2$, «Lessing », Avril-Juin 2003, p. 167.

7. «Schwärmerei ist Krankheit der Seele, eigentliches Seelenfiebes: Enthusiasmus ist ihr wahres Leben! - Welch ein Unterschied. » (Der teutsche Merkur, ouvr. cité, p. 153)

8. Ibid., p. 134.

9. Wieland, Sämtliche Werke, Hamburg, 1984, Bd. 24/VIII, p. 92. 
10. «Es scheint seltsam, daß zwei so widersprechende Neigungen als der Hang zum Wunderbaren und die Liebe zum Wahren dem Menschen gleichgütlig, gleich wesentlich sein sollen und doch ist es nicht anders. » (Dschinnistan, Vorrede)

11. Shaftesbury faisait déjà de "good Humor» ce qu'il appelait «the best security against enthusiasm ".

12. Veteres avias: les vieux préjugés. Dum veteres avias tibi de pulmone revello (Perse, cinquième satire, v. 92. "Les préjugés reçus de nos nourrices, de nos grand-mères; l'ignorance du premier âge de la raison que nous regrettons quelque fois", Satires de Perse, par A. Desportes, Paris, Hachette, 1841, p. 239). (C. M. Wieland, Don Sylvio, « Supplément de l'éditeur »).

13. C. M. Wieland, Don Sylvio, Premier livre, chap. 3.

14. Auswahl denkwürdiger Briefe von C. M. Wieland, Wien, C. Gerold, 1815, vol. I, p. 6.

15. V. Propp, Morphologie du conte, Paris, Seuil/Points, 1965.

16. C. M. Wieland, Don Sylvio, Cinquième livre, chap. 1.

17. C. M. Wieland, Don Sylvio, Deuxième livre, chap. 6.

18. Condillac, Traité des sensations [1754], Paris, Fayard, 1984, p. 147.

19. A. G. Meusnier de Querlon, Les Soupers de Daphene et les dortoirs de Lacédémone, anecdotes grecques ou fragments historiques, Oxford, 1740.

20. «Sa peau sèche et détendue reprend le poli, la fraîcheur et la molle fermeté des plus belles chairs, les cuisses toutes décharnées s'arrondissent, et le squelette entier devient un corps revêtu de cet embonpoint délicat qui est l'apanage de la jeunesse. Tel est l'enchantement du beau Glycon. » A. G. Meusnier de Querlon, Les Dortoirs de Lacédémone, éd. M. Tourneux, Paris, Librairie des Bibliophiles, 1886, p. 62.

21. C. M. Wieland, Don Sylvio, Troisième livre, chap. 9. On voit par là que l'illusion n'est pas celle des sens, mais bien de l'interprétation que l'on fait des choses perçues.

22. C. M. Wieland, Don Sylvio, Cinquième livre, chap. 1.

23. C. M. Wieland, Don Sylvio, Premier livre, chap. 5.

24. «Il prit les mesures les plus prudentes pour que sa tante, qui était sévère et un peu rusée, ne découvrît pas les raisons qu'il avoir de se promener si souvent dans le bois. Si elle s'était aperçue de quelque chose, il est certain qu'elle lui aurait fait de très fortes, de très savantes et de très ennuyeuses représentations. "

25. C. M. Wieland, Don Sylvio, Première partie, chap. 4.

26. C. M. Wieland, Don Sylvio, Premier livre, chap. 4.

27. C. M. Wieland, Don Sylvio, Premier livre, chap. 3.

28. É. Jacquelin, « Rêves et visions dans Les Aventures de Don Sylvio de Rosalva (C. M. Wieland, 1764), ou Le laboratoire du songe-creux ", dans M. Demaules, Expériences oniriques dans la littérature et les arts du Moyen Âge au XVIII ${ }^{e}$ siècle, Paris, H. Champion, 2016, p. 362.

29. Ibid., p. 363.

30. Wieland se souvient de Charles Sorel: «Ma foi, ce dit alors Francion, il me semble que j'entends encore les fables de ces fées, dont les servantes entretiennent les enfans. L'on dit que, si elles alloient à la selle, elles n'y faisoient que du musc; si elles pissoient, c'étoit eau d'ange ; si elles crachoient ou si elles se mouchoient, il sortoit de leur nez et de leur bouche des émeraudes et des perles; et, si elles lavoient leurs mains, au lieu de crasse il en tomboit aussi des pierres précieuses. » La Vraie Histoire comique de Francion [1623], A. Delahays, 1858, liv. 11, p. 438.

31. Fée lumineuse qu'on trouve aussi bien dans L'Heureuse peine de la Comtesse de Murat que chez le comte de Caylus.

32. Arménis a «cette tournure de Cour frivole et légère qui est si à la mode aujourd'hui : en un mot, elle est excellente pour former un jeune homme " (La Morlière, Angola, histoire indienne, éd. J.-P. Sermain, Paris, Desjonquères, 1992, p. 113).

33. Ibid., p. 91.

34. Ibid., p. 77.

Féeries, 15 | 2018 
35. Ibid., p. 70.

36. C. M. Wieland, Don Sylvio, Sixième livre, chap. 2.

37. C. M. Wieland, Don Sylvio, Sixième livre, chap. 1.

38. Ibid.

39. M. A. von Thümmel, Die Inoculation der Liebe, Leipzig, Weidmanns Erben und Reich, 1771, p. 7.

40. Wieland à Julie Bondeli, 16 juillet 1764 [nous citons là le français de Wieland !].

41. Qui sera critiquée par les romantiques. Il est vrai du reste que le début de Don Sylvio semble être une parodie avant la lettre de Heinrich von Ofterdingen (1802) tant les choses se sont inversées. 42. C. M. Wieland, Don Sylvio, Sixième livre, chap. 2.

43. «Ich nenne [...] Schwärmerey eine Erhitzung der Seele von Gegenständen die entweder gar nicht in die Natur sind, worüber die betauschte Seele sie ansieht. » (Der teutsche Merkur, ouvr. cité, p. 152)

44. Auswahl denkwürdiger Briefe von C. M. Wieland, ouvr. cité, p. 5.

\section{RÉSUMÉS}

Le titre même du roman de Wieland intitulé Le triomphe de la nature sur la rêverie ou les aventures de Don Sylvio de Rosalva indique le conflit fondamental de l'imagination et de la réalité objective. Il présente un héros qui transpose en imagination ses perceptions du réel et interprète le monde suivant les contes de fées dont il est friand lecteur. Pour le désenchanter ses amis lui racontent un conte merveilleux, le conte de Biribinker, dont l'extravagance est destinée à le ramener à la saine réalité. Le jeu de l'illusion et de ses ruptures, la critique de la superstition, les rapports du perçu et de l'imaginé posent de manière moderne le rapport des mondes possibles et du réel.

The very title of Wieland's novel, The Triumph of Nature on Reverie or the Adventures of Don Sylvio de Rosalva, indicates the fundamental conflict of imagination and objective reality. The author presents a hero, who transposes his perceptions of the real into imagination and interprets the world according to the fairy tales of which he is a fond reader. To disillusion him, his friends recount a wonderful tale, the tale of Biribinker, whose extravagance is intended to healthily bring him back to reality. The play of illusion and its breaks, the criticism of superstition, the relations between the perceived and the imagined establish a modern relation between possible worlds and reality.

\section{INDEX}

Mots-clés : contes de fées, Wieland, rêverie, imagination, délire, superstition, illusion

Keywords : fairy tales, Wieland, reverie, imagination, delirium, superstition, illusion

\section{AUTEUR}

\section{ALAIN MONTANDON}

Université Clermont Auvergne, CELIS 